\title{
A NOTE ON METRIC INHOMOGENEOUS DIOPHANTINE APPROXIMATION
}

\author{
M. M. DODSON \\ (Received 25 August 1995; revised 25 January 1996)
}

Communicated by W. W. L. Chen

\begin{abstract}
An inhomogeneous version of a general form of the Jarnik-Besicovitch Theorem is proved.

1991 Mathematics subject classification (Amer. Math. Soc.): primary 11J83; secondary $11 \mathrm{~J} 20$.
\end{abstract}

Dedicated to Professor F. Chong for his 80th birthday

\section{Introduction}

In some respects inhomogeneous Diophantine approximation is rather different from homogeneous Diophantine approximation. Results in the former, where the additional variables offer extra 'degrees of freedom', are sometimes sharper or easier to prove than the corresponding ones in the latter. For example, if the real numbers $x, \alpha$ do not satisfy $\alpha=k x+l$ for any integers $k, l$, then

$$
|q|\|q x-\alpha\|<1 / 4
$$

for infinitely many integers $q$ (see [5, Theorem IIA, Chapter 3]). Here

$$
\|x\|=\min \{|x-k|: k \in \mathbb{Z}\}=\min \{\{x\}, 1-\{x\}\},
$$

where $\{x\}$ is the fractional part of $x$. However if $x$ is irrational, the inequality

$$
|q|\|q x\|<1 / \sqrt{5}
$$

(C) 1997 Australian Mathematical Society 0263-6115/97 \$A2.00+0.00 
holds for infinitely many integers $q$ (see [5, Theorem $\mathrm{V}$ in Chapter 1]). Both these results are best possible. Again, an inhomogeneous version of Khintchine's theorem on simultaneous Diophantine approximation (see [5, Theorem II in Chapter 7]) is not only considerably easier to prove but is also slightly more general than the homogeneous case ([5, Theorem I in Chapter 7]). In this version, the result holds for 'almost all' the additional points as well and is thus 'doubly metric' [17]. The same is true for the more general Khintchine-Groshev Theorem [18, Chapter 1, Theorem 12] as the inhomogeneous 'doubly metric' case has a much simpler proof.

On the other hand the extra variables can 'interfere' with the homogeneous variables; for this reason there is no inhomogeneous counterpart to Dirichlet's theorem (see [5, Theorem III in Chapter 3]). Also, the proof of the inhomogeneous KhintchineGroshev theorem, where the additional variables are fixed, requires a little more work (it is a special case of [18, Chapter 1 Theorem 15]) and Schmidt described his extension [17] of his quantitative refinement [16] of Khintchine's theorem to a result implying the inhomogeneous case as 'non-trivial'.

The purpose of this note is to establish an inhomogeneous counterpart of a more general form [6] of the Jarník-Besicovitch theorem [3, 12]. Firstly some simplifying notation is introduced. For each real number $\alpha$, let

$$
\langle\alpha\rangle=\left\{\begin{array}{lll}
\{\alpha\} & \text { when } & \{\alpha\} \leq 1 / 2 \\
-1+\{\alpha\} & \text { when } & 1 / 2<\{\alpha\}<1
\end{array}\right.
$$

denote the symmetrised fractional part of $\alpha$, which is $\alpha$ translated by a unique integer $k_{\alpha}$ to $(-1 / 2,1 / 2]$. Clearly $|\langle\alpha\rangle|=\|\alpha\|$. In higher dimensions, $\langle\alpha\rangle$ will denote the symmetrical fractional part of the vector $\alpha \in \mathbb{R}^{n}$, that is,

$$
\langle\alpha\rangle=\left(\left\langle\alpha_{1}\right\rangle, \ldots,\left\langle\alpha_{n}\right\rangle\right)=\alpha+\boldsymbol{k}_{\alpha} \in(-1 / 2,1 / 2]^{n}
$$

for a unique $\boldsymbol{k}_{\boldsymbol{\alpha}} \in \mathbb{Z}^{n}$. For each vector $\boldsymbol{v}=\left(v_{1}, \ldots, v_{n}\right)$, write

$$
|\boldsymbol{v}|=|\boldsymbol{v}|_{\infty}=\max \left\{\left|v_{j}\right|: j=1, \ldots, n\right\}
$$

for the sup norm of $v$. The Euclidean norm of $v$ will be written $|v|_{2}$.

Let $V(\psi)$ denote the set of points $(X, \alpha) \in \mathbb{R}^{m n} \times \mathbb{R}^{n}$ for which the system of inequalities given by

$$
|\langle\boldsymbol{q} X-\boldsymbol{\alpha}\rangle|=\max _{j=1, \ldots, n}\left\|\sum_{i=1}^{m} q_{i} x_{i j}-\alpha_{j}\right\|<\psi(|\boldsymbol{q}|),
$$

where $\left(x_{i j}\right)=X$, has infinitely many solutions $q \in \mathbb{Z}^{m}$. The Hausdorff dimension $\operatorname{dim} V(\psi)$ of $V(\psi)$ is obtained in terms of the lower order (at infinity) of the positive function $1 / \psi$ (the lower order $\lambda(f)$ of a positive function $f: \mathbb{N} \rightarrow \mathbb{B}^{+}$is defined by $\left.\lambda(f)=\liminf _{q \rightarrow \infty}(\log f(q)) /(\log q)\right)$. 
THEOREM. Let $\psi$ be a decreasing positive function and let $\lambda$ be the lower order of $1 / \psi$. Then

$$
\operatorname{dim} V(\psi)= \begin{cases}m n+(m+n) /(\lambda+1) & \text { when } \lambda \geq m / n \\ (m+1) n & \text { when } \lambda \leq m / n\end{cases}
$$

The natural precursor (mentioned above) of this result is the 'doubly metric' inhomogeneous version of the Khintchine-Groshev theorem, which asserts that for any positive sequence $\psi(r), r=1,2, \ldots$, the Lebesgue measure $|V(\psi)|$ of $V(\psi)$ is given by

$$
|V(\psi)|=\left\{\begin{array}{lll}
0 & \text { when } & \sum_{r=1}^{\infty} r^{m-1} \psi(r)^{n}<\infty \\
1 & \text { when } & \sum_{r=1}^{\infty} r^{m-1} \psi(r)^{n}=\infty .
\end{array}\right.
$$

It is 'doubly metric' in the sense that it holds for 'almost all' $X$ and $\alpha$ and is an immediate consequence of [17, Theorem 1] or [18, Chapter 1, Theorem 15]. However in contrast with the (homogeneous) Khintchine-Groshev Theorem, this version also has a simple direct proof since some of the complications in the homogeneous case do not arise $[7,18]$. Note that the sequence $\psi(r), r=1,2, \ldots$, is not required to decrease monotonically.

In this more general setting, the inhomogeneous case can be reduced to a special case of the homogeneous case (where $\boldsymbol{\alpha}=\mathbf{0}$ ) by considering the vectors $\boldsymbol{q} \in \mathbb{Z}^{m+1}$ restricted to $\mathbb{Z}^{m} \times\{-1\}$. However the proofs of the homogeneous case in $[6,7$, 15] either cannot be adapted or they involve complications which are not relevant to inhomogeneous Diophantine approximation.

(Added in proof. H. Dickinson has proved the above theorem using restricted Diophantine approximation in the paper 'A remark on a theorem of Jarník', to appear in Glasgow Math. J.)

Let $I=[0,1]$. Since $(X, \boldsymbol{\alpha}) \mapsto\langle q X-\boldsymbol{\alpha}\rangle$ is 1-periodic, there is no loss in generality in restricting $X$ to $I^{m n}$ and $\alpha$ to $I^{n}$. In addition, $M_{m \times n}(I)$ will be identified with $I^{m n}$. Write $\Omega=I^{m n} \times I^{n}$. Then the set $V(\psi)$ can be expressed as the 'lim-sup' set

$$
V(\psi)=\bigcap_{N=1}^{\infty} \bigcup_{|q|=N}^{\infty} B_{\psi(|q|)}\left(R_{q}\right)
$$

where

$$
R_{q}=\{(X, \boldsymbol{\alpha}) \in \Omega:\langle q X-\boldsymbol{\alpha}\rangle=\mathbf{0}\},
$$

and for each positive $\delta$

$$
B_{\delta}\left(R_{q}\right)=\{(X, \boldsymbol{\alpha}) \in \Omega:|\langle\boldsymbol{q} X-\boldsymbol{\alpha}\rangle|<\delta\}
$$


is a neighbourhood of $R_{q}$. Later, the related neighbourhood $B\left(R_{q} ; \delta\right)$ given by

$$
B\left(R_{q} ; \delta\right)=\left\{(X, \alpha) \in \Omega: \operatorname{dist}_{\infty}\left((X, \alpha), R_{q}\right)<\delta\right\}
$$

where $\left.\operatorname{dist}_{\infty}(\omega, A)=\inf \{|\omega-a|: a \in A)\right\}$ is the distance in the supremum metric of $\omega$ from the set $A$, will be used.

\section{Volume calculations}

Let $0<\delta<1 / 2$ and let $\chi_{S}$ be the characteristic function on the set $S$. For each $X \in I^{m n}$ and $q \in \mathbb{Z}^{m}$, the substitution $\alpha^{\prime}=\alpha-q X$ gives

$$
\left|\left\{\boldsymbol{\alpha} \in I^{n}:|\langle\boldsymbol{q} X-\boldsymbol{\alpha}\rangle|<\delta\right\}\right|=\int_{I^{n}} \chi_{(-\delta, \delta)^{n}}(\langle\boldsymbol{q} X-\boldsymbol{\alpha}\rangle) d \boldsymbol{\alpha}=2^{n} \delta^{n} .
$$

Hence the Lebesgue measure $\left|B_{\delta}(R(q))\right|$ of $B_{\delta}(R(q))$ is given by

$$
\begin{aligned}
\left|B_{\delta}(R(\boldsymbol{q}))\right| & =\int_{I^{m n}} \int_{I^{n}} \chi_{B_{\delta}\left(R_{q}\right)}(X, \boldsymbol{\alpha}) d \boldsymbol{\alpha} d X \\
& =\int_{I^{m n}} \int_{I^{n}} \chi_{(-\delta, \delta)^{n}}(\langle\boldsymbol{q} X-\boldsymbol{\alpha}\rangle) d \boldsymbol{\alpha} d X=2^{n} \delta^{n} .
\end{aligned}
$$

Note that given $\alpha$ and a non-zero $q$, the 1-periodicity of the function

$$
\chi_{B_{\delta}\left(R_{q}\right)}(X, \boldsymbol{\alpha})=\chi_{(-\delta, \delta)^{n}}(\langle\boldsymbol{q} X-\boldsymbol{\alpha}\rangle)
$$

together with some geometry $[6,10]$ or Fourier analysis $[18$, Lemma 8 , p. 35] give

$$
\left|\left\{X \in I^{m n}:|\langle\boldsymbol{q} X-\boldsymbol{\alpha}\rangle|<\delta\right\}\right|=\int_{I^{m n}} \chi_{(-\delta, \delta)^{n}}(\langle\boldsymbol{q} X-\boldsymbol{\alpha}\rangle) d X=2^{n} \delta^{n}
$$

(see $[6,10]$ and $[18$, Lemma 8, p. 35]).

LEMMA 1. Let $\delta, \eta \in(0,1 / 2)$ and let $\boldsymbol{q}, \boldsymbol{r}$ be distinct non-zero vectors in $\mathbb{Z}^{m}$. Then

$$
\left|B_{\delta}\left(R_{q}\right) \cap B_{\eta}\left(R_{r}\right)\right|=\left|B_{\delta}\left(R_{q}\right)\right|\left|B_{\eta}\left(R_{r}\right)\right|=4^{n} \delta^{n} \eta^{n} .
$$

PROOF. On making the substitution $\boldsymbol{\alpha}^{\prime}=\boldsymbol{\alpha}-\boldsymbol{q} X$ and integrating, it follows from the above that

$$
\begin{aligned}
\left|B_{\delta}\left(R_{\boldsymbol{q}}\right) \cap B_{\eta}\left(R_{\boldsymbol{r}}\right)\right| & =\int_{I^{m n}} \int_{I^{n}} \chi_{(-\delta, \delta)^{n}}(\langle\boldsymbol{q} X-\boldsymbol{\alpha}\rangle) \chi_{(-\eta, \eta)^{n}}(\langle\boldsymbol{r} X-\boldsymbol{\alpha}\rangle) d \boldsymbol{\alpha} d X \\
& =\int_{I^{n}} \chi_{(-\delta, \delta)^{n}}\left(\left\langle\boldsymbol{\alpha}^{\prime}\right\rangle\right)\left(\int_{I^{m n}} \chi_{(-\eta, \eta)^{n}}\left(\left\langle(\boldsymbol{r}-\boldsymbol{q}) X-\boldsymbol{\alpha}^{\prime}\right\rangle\right) d X\right) d \boldsymbol{\alpha}^{\prime} \\
& =\int_{I^{n}} \chi_{(-\delta, \delta)^{n}}\left(\left\langle\boldsymbol{\alpha}^{\prime}\right\rangle\right)\left(2^{n} \eta^{n}\right) d \boldsymbol{\alpha}^{\prime}=4^{n} \delta^{n} \eta^{n}
\end{aligned}
$$

This lemma does not hold in the homogeneous case when $m=1$, corresponding to simultaneous Diophantine approximation (see $[5,18]$ ). 


\section{An inhomogeneous Jarník-Besicovitch theorem}

By contrast with the inhomogeneous Khintchine-Groshev theorem, the "doubly metric' inhomogeneous Jarník-Besicovitch theorem which gives the Hausdorff dimension of $V(\psi)$ is quite difficult; as is often the case, this difficulty lies in establishing the correct lower bound. Dirichlet's theorem and the more general linear forms theorem of Minkowski do not hold in the inhomogeneous setting and so cannot be used, as they can be to prove the Jarník-Besicovitch theorem and its generalisations $[2,6,8]$. In the case of simultaneous Diophantine approximation, Jarník [13] used a Cantor-type construction to determine the Hausdorff $s$-measure; other approaches are given in $[4,7,11]$. A. Baker and W. M. Schmidt introduced regular systems to obtain lower bounds for the Hausdorff dimension of certain subsets of the line to generalise the Jarník-Besicovitch theorem to approximation by real algebraic numbers [2]. In order to study geodesic excursions in hyperbolic manifolds, Melián and Pestana [14] extended regular systems to 'well-distributed' systems in higher dimensions. However this approach is limited to approximating points in $\mathbb{R}^{k}$ and is not suitable for inhomogeneous Diophantine approximation, nor for approximation questions arising from the problem of 'resonant' sets or 'small denominators', associated with stability or normal forms questions. To deal with these rather varied questions, the more general notion of ubiquity has been introduced (for details see [8] and for some applications, see [9]). As usual the proof of the theorem is in two parts, with the upward and downward inequalities for the Hausdorff dimension being dealt with separately.

The upward inequality in the theorem is straightforward but is included for completeness (note that $\psi$ need not be decreasing).

LEMMA 2. Let $\psi$ be a positive function and let $\lambda$ be the lower order of $1 / \psi$. Then

$$
\operatorname{dim} V(\psi) \leq \begin{cases}m n+(m+n) /(\lambda+1) & \text { when } \lambda \geq m / n \\ m n+n & \text { when } \lambda \leq m / n\end{cases}
$$

PROOF. Since $V(\psi) \subset I^{m n} \times I^{n}, \operatorname{dim} V(\psi) \leq(m+1) n$. The other part of the bound is obtained by constructing a suitable cover from (2). For each $N=1,2, \ldots$, the collection

$$
\left\{B_{\psi(|q|)}\left(R_{q}\right): q \in \mathbb{Z}^{m},|\boldsymbol{q}| \geq N\right\}
$$

is a cover of $V(\psi)$. For each non-zero $q \in \mathbb{Z}^{m}, B_{\psi(|q|)}\left(R_{q}\right)$ is covered by a collection $\mathscr{C}(\boldsymbol{q})$ say of at most

$$
\ll\left(\frac{\psi(|\boldsymbol{q}|)}{|\boldsymbol{q}|}\right)^{-m n}|\boldsymbol{q}|^{n} \ll|\boldsymbol{q}|^{(m+1) n} \psi(|\boldsymbol{q}|)^{-m n}
$$


(mn $+n$ )-dimensional hypercubes $C$ of sidelength $\ell(C)=4 \psi(|q|) /|q|$ and with centres on the $m n$-dimensional (resonant) set (a finite union of hyperplanes) $R_{q}$ at integral multiples of $\psi(|\boldsymbol{q}|) /|\boldsymbol{q}|$ apart on the hyperplanes $R_{q, r}$. Thus for each $N=$ $1,2, \ldots$, the collection $\mathscr{C}_{N}=\{C \in \mathscr{C}(\boldsymbol{q}):|\boldsymbol{q}| \geq N\}$ of hypercubes $C$ with $\ell(C) \ll$ $\psi(N) / N$ (since $\psi$ is decreasing) is a cover of $V(\psi)$. Moreover the $s$-length $\ell^{s}\left(\mathscr{C}_{N}\right)$ of the cover $\mathscr{C}_{N}$ satisfies

$$
\ell^{s}\left(\mathscr{C}_{N}\right) \leq 4^{s} \sum_{q \geq N} \sum_{|q|=q}|q|^{(m+1) n} \psi(|q|)^{-m n}(\psi(|q|) /|q|)^{s} .
$$

From the definition of lower order, given any $\varepsilon>0, \psi(q) \ll q^{-\lambda+\varepsilon}$, whence for $s>m n+(m+n) /(\lambda+1)=s-\eta$ say, where $\eta>0$,

$$
\begin{aligned}
\ell^{s}\left(\mathscr{C}_{N}\right) & \ll \sum_{q=N}^{\infty} q^{m-1} q^{m n+n} q^{(-\lambda+\varepsilon)(s-m n)} q^{-s} \\
& \ll \sum_{q=N}^{\infty} q^{m+n+m n(\lambda+1)-(\lambda+1) s-1+\varepsilon(s-m n)} \\
& \ll \sum_{q=N}^{\infty} q^{-1-\varepsilon^{\prime}}
\end{aligned}
$$

where $\varepsilon^{\prime}=(\lambda+1) \eta-\varepsilon(s-m n)>0$ for $\varepsilon$ sufficiently small. Thus $\ell^{s}\left(\mathscr{C}_{N}\right) \rightarrow 0$ as $N \rightarrow \infty$ and the result follows from the definition of Hausdorff dimension.

The harder downward inequality is obtained by using the idea of ubiquity [8] combined with a variance argument, an approach which has been taken in [7] for homogeneous Diophantine approximation .

Let $S$ be an open non-empty subset of Euclidean space. Let $\mathscr{R}=\left\{R_{j}: j \in J\right\}$ where $J$ is a countable index set and where the $R_{j}$ are finite unions of affine $d$-dimensional proper subspaces of Euclidean space intersected with $S$. Suppose each $j \in J$ has a positive weight $\lfloor j\rfloor$. The sets $R_{j}$ are called resonant sets and their common dimension $d$ will be written $\operatorname{dim} \mathscr{R}$ and their codimension $\operatorname{dim} S-\operatorname{dim} \mathscr{R}$ written $\operatorname{codim} \mathscr{R}$. Let $(\varphi(q): q=1,2, \ldots)$ be a decreasing sequence with $\varphi(q) \rightarrow 0$ as $q \rightarrow \infty$. The lim-sup set

$$
\Lambda(\mathscr{R} ; \varphi)=\left\{\omega \in S: \operatorname{dist}\left(\omega, R_{j}\right)<\varphi(\lfloor j\rfloor) \text { for infinitely many } j \in J\right\},
$$

where $\operatorname{dist}(\omega, A)$ is the distance of $\omega \in S$ from the set $A$ in the sup norm, consists of points in $S$ which are ' $\varphi$-approximable' by $\mathscr{R}$ in the sup norm. A lower bound for the Hausdorff dimension of such sets can be obtained when the family $\mathscr{R}$ of resonant sets is ubiquitous in the following sense. Let $\rho: \mathbb{N} \rightarrow \mathbb{R}^{+}$be a function converging to 0 at infinity. Then $\mathscr{R}$ is ubiquitous with respect to $\rho$ if

$$
\left|S \backslash \bigcup_{\lfloor j\rfloor \leq N} B\left(R_{j} ; \rho(N)\right)\right| \rightarrow 0 \quad \text { as } \quad N \rightarrow \infty,
$$


where for any subset $A, B(A ; \delta)=\left\{\omega \in S\right.$ : $\left.\operatorname{dist}_{\infty}(\omega, A)<\delta\right\}$. This formulation is equivalent for affine spaces to the more general definition in [8]. It is shown there that if $\mathscr{R}$ is ubiquitous with respect to $\rho$, then

$$
\operatorname{dim} \Lambda(\mathscr{R} ; \varphi) \geq \operatorname{dim} \mathscr{R}+\gamma \operatorname{codim} \mathscr{R},
$$

where

$$
\gamma=\min \left\{1, \limsup _{N \rightarrow \infty} \frac{\log \rho(N)}{\log \varphi(N)}\right\} .
$$

To apply this result to $V(\psi)$, take $S=\Omega=I^{m n} \times I^{n}, J=\mathbb{Z}^{m} \backslash\{0\}, j=\boldsymbol{q}$, $\lfloor q\rfloor=|q|$ and take the resonant sets $R_{q}$ to be given by (3), that is,

$$
R_{q}=\{(X, \alpha) \in \Omega:\langle q X-\alpha\rangle=0\} .
$$

Thus the resonant sets have dimension $m n$ and codimension $n$. With these choices, the set $\Lambda(\mathscr{R} ; \varphi)$ of points $(X, \alpha) \in \Omega$ such that dist $\left((X, \alpha), R_{q}\right)<\varphi(|q|)$ for infinitely many $q \in \mathbb{Z}^{m}$ becomes

$$
\Lambda(\mathscr{R} ; \varphi)=\bigcap_{N=1}^{\infty} \bigcup_{|q|=N}^{\infty} B\left(R_{q} ; \varphi(|q|)\right) .
$$

Now (see (3)), $R_{q}=\bigcup_{r} R_{q, r}$ where the union is over those $r \in\left(q I^{m n}\right) \cap \mathbb{Z}^{n}$ (recall that $I^{m n}$ is identified with $\left.M_{m \times n}(I)\right)$. Each $R_{q, r}=\{(X, \alpha) \in \Omega: q X-\alpha=r\}=$ $\left(R_{q, r_{1}}, \ldots, R_{q, r_{n}}\right)$, where

$$
R_{q, r_{j}}=\left\{\left(\boldsymbol{x}^{(j)}, \alpha_{j}\right): \boldsymbol{q} \cdot \boldsymbol{x}^{(j)}-\alpha_{j}=r_{j}\right\}
$$

and $x^{(j)}$ is the $j$ th column of $X$.

Let $\tilde{\boldsymbol{q}}=(\boldsymbol{q},-1)$ and let $\tilde{X}$ denote the $(m+1) \times n$ matrix given by $X$ with $\alpha$ added as the $(m+1)$ th row. Write the $j$ th column of $\widetilde{X}$ as $\widetilde{\boldsymbol{x}}^{(j)}$. When $\boldsymbol{q} \neq 0$,

$$
\begin{aligned}
\operatorname{dist}_{\infty}\left(\tilde{X}, R_{q}\right) & =\min \left\{\operatorname{dist}_{\infty}\left(\tilde{X}, R_{q, r}\right): r \in\left(q I^{m n}\right) \cap \mathbb{Z}^{n}\right\} \\
& =\min _{r}\left\{\min \left\{\operatorname{dist}_{\infty}\left(\widetilde{\boldsymbol{x}}^{(j)}, \boldsymbol{R}_{\boldsymbol{q}, r_{j}}\right): 1 \leq j \leq n\right\}\right\}
\end{aligned}
$$

and for some $j_{0}, 1 \leq j_{0} \leq n$ and $\widetilde{\mathbf{y}}$ lying in $R_{\boldsymbol{q}, r_{j_{0}}}$ (so that $\tilde{\boldsymbol{q}} \cdot \widetilde{\mathbf{y}}=r_{j_{0}}$ )

$$
|\langle\tilde{\boldsymbol{q}} \tilde{X}\rangle|=\max \left\{\left|\left\langle\tilde{\boldsymbol{q}} \cdot \tilde{\boldsymbol{x}}^{(j)}\right\rangle\right|: 1 \leq j \leq n\right\}=\left|\left\langle\tilde{\boldsymbol{q}} \cdot \tilde{\boldsymbol{x}}^{\left(j_{0}\right)}\right\rangle\right|=\left|\tilde{\boldsymbol{q}} \cdot\left(\tilde{\boldsymbol{x}}^{\left(j_{0}\right)}-\tilde{\boldsymbol{y}}\right)\right| .
$$

Suppose $\operatorname{dist}_{\infty}\left(\tilde{X}, R_{\boldsymbol{q}}\right)<\delta /(m+1)|\tilde{\boldsymbol{q}}|$. Then

$$
|\langle\tilde{\boldsymbol{q}} \tilde{X}\rangle| \leq(m+1)|\tilde{\boldsymbol{q}}|\left|\tilde{\boldsymbol{x}}^{\left(j_{0}\right)}-\tilde{\boldsymbol{y}}\right| \leq(m+1)|\tilde{\boldsymbol{q}}| \delta /((m+1)|\tilde{\boldsymbol{q}}|)=\delta .
$$


If $|\langle\tilde{\boldsymbol{q}} \tilde{X}\rangle|<\delta$, then

$$
\begin{aligned}
\operatorname{dist}_{\infty}\left(\tilde{X}, R_{q}\right) & \leq \min _{r} \min \left\{\operatorname{dist}_{2}\left(\tilde{\boldsymbol{x}}^{(j)}, R_{\boldsymbol{q}, r_{j}}\right): 1 \leq j \leq n\right\} \\
& =\min _{\boldsymbol{r}} \min \left\{\left|\tilde{\boldsymbol{q}} \cdot \tilde{\boldsymbol{x}}^{(j)}-r_{j}\right| /|\tilde{\boldsymbol{q}}|_{2}: 1 \leq j \leq n\right\} \\
& \leq \delta /|\tilde{\boldsymbol{q}}| .
\end{aligned}
$$

Thus when $\tilde{\boldsymbol{q}} \neq \mathbf{0}$, the neighbourhoods $B_{\delta}\left(R_{q}\right)$ and $B\left(R_{q} ; \delta\right)$ satisfy

$$
B\left(R_{q} ; \delta /(m+1)|\tilde{q}|\right) \subseteq B_{\delta}\left(R_{q}\right) \subseteq B\left(R_{q} ; \delta /|\tilde{q}|\right)
$$

But $\boldsymbol{q} \neq 0$ implies that $|\tilde{\boldsymbol{q}}|=|\boldsymbol{q}|$, whence for $\boldsymbol{q} \neq \mathbf{0}$

$$
B\left(R_{q} ; \delta /(m+1)|q|\right) \subseteq B_{\delta}\left(R_{q}\right) \subseteq B\left(R_{q} ; \delta /|q|\right)
$$

By (2) and (5), it follows that $\Lambda(\mathscr{R}, \varphi) \subseteq V(\psi)$ when $\varphi(q)=\psi(q) /(m+1) q$. As well, the family $\mathscr{R}$ of resonant sets is ubiquitous with respect to the function $\rho$ given by

$$
\rho(N)=N^{-1-m / n} \log N,
$$

as is now shown.

LEMMA 3. The family $\mathscr{R}=\left\{R_{q}: q \in \mathbb{Z}^{m} \backslash\{0\}\right\}$ of resonant sets $R_{q}$ is ubiquitous with respect to the function $\rho$ given by (8).

PROOF. It has to be shown that

$$
\left|\Omega \backslash \bigcup_{1 \leq|q| \leq N} B\left(R_{q} ; \rho(N)\right)\right| \rightarrow 0 \quad \text { as } \quad N \rightarrow \infty .
$$

For each $\boldsymbol{q}$ and $N$, let $\tilde{\rho}(q)=\rho(N)|q|$. By (7), $B_{\tilde{\rho}(q)}\left(R_{q}\right) \subseteq B\left(R_{q} ; \rho(N)\right)$, and so it suffices to show that

$$
\left|\Omega \backslash \bigcup_{1 \leq|q| \leq N} B_{\tilde{\rho}(q)}\left(R_{q}\right)\right| \rightarrow 0 \text { as } N \rightarrow \infty
$$

Now let

$$
v_{N}(X, \boldsymbol{\alpha})=\sum_{\mathbf{l} \leq|\boldsymbol{q}| \leq N} \chi_{(-\tilde{\rho}(\boldsymbol{q}), \bar{\rho}(\boldsymbol{q}))^{n}}(\langle\boldsymbol{q} X-\boldsymbol{\alpha}\rangle) .
$$

Thus $v_{N}(X, \boldsymbol{\alpha})$ is the number of resonant sets $R_{q}, 1 \leq|\boldsymbol{q}| \leq N$ such that the point $(X, \alpha)$ lies in the neighbourhood $B_{\tilde{\rho}(q)}\left(R_{q}\right)$ of $R_{q}$. Further, $\nu_{N}^{-1}(0)$ is the set of points $(X, \alpha) \in \Omega$ such that

$$
|\langle\boldsymbol{q} X-\boldsymbol{\alpha}\rangle| \geq \tilde{\rho}(\boldsymbol{q})
$$

holds for all $q \in \mathbb{Z}^{m}$ with $1 \leq|q| \leq N$, that is,

$$
v_{N}^{-1}(0)=\Omega \backslash \bigcup_{1 \leq|q| \leq N} B_{\tilde{\rho}(q)}\left(R_{q}\right)
$$


Now let $\mu_{N}$ be the mean of $v_{N}$. Then by (4),

$$
\begin{aligned}
\mu_{N} & =\int_{\Omega} v_{N}(X, \boldsymbol{\alpha}) d X d \boldsymbol{\alpha}=\int_{I^{m n}} \int_{I^{n}} \sum_{1 \leq|\boldsymbol{q}| \leq N} \chi_{B_{\bar{\rho}(q)}\left(R_{q}\right)}(X, \boldsymbol{\alpha}) d X d \boldsymbol{\alpha} \\
& =\sum_{1 \leq|\boldsymbol{q}| \leq N}\left|B_{\tilde{\rho}(\boldsymbol{q})}\left(R_{\boldsymbol{q}}\right)\right|=2^{n} \rho(N)^{n} \sum_{1 \leq|\boldsymbol{q}| \leq N}|\boldsymbol{q}|^{n} \\
& =2^{n} \rho(N)^{n} \sum_{1 \leq q \leq N} q^{n} \sum_{|q|=q} 1 \asymp \rho(N)^{n} \sum_{1 \leq q \leq N} q^{n+m-1} \\
& \asymp \rho(N)^{n} N^{m+n} \rightarrow \infty
\end{aligned}
$$

as $N \rightarrow \infty$ by (8) ( $a \asymp b$ when $a \ll b \ll a)$. The variance $\sigma_{N}^{2}$ of $\mu_{N}$ is given by

$$
\begin{aligned}
\sigma_{N}^{2} & =\int_{\Omega}\left(v_{N}(X, \boldsymbol{\alpha})-\mu_{N}\right)^{2} d X d \boldsymbol{\alpha}=\int_{\Omega} v_{N}(X, \boldsymbol{\alpha})^{2} d X d \boldsymbol{\alpha}-\mu_{N}^{2} \\
& =\sum_{1 \leq|\boldsymbol{q}| \leq N} \sum_{1 \leq|r| \leq N} \int_{\Omega} \chi_{B_{\bar{\rho}(\boldsymbol{q})}\left(R_{\boldsymbol{q}}\right)}(X, \boldsymbol{\alpha}) \chi_{B_{\tilde{\rho}(r)}\left(R_{r}\right)}(X, \boldsymbol{\alpha}) d X d \boldsymbol{\alpha}-\mu_{N}^{2} \\
& =\sum_{1 \leq|\boldsymbol{q}| \leq N}\left|B_{\tilde{\rho}(\boldsymbol{q})}\left(R_{\boldsymbol{q}}\right)\right|+\sum_{\boldsymbol{q} \neq \boldsymbol{r}}\left|B_{\tilde{\rho}(\boldsymbol{q})}\left(R_{\boldsymbol{q}}\right) \cap B_{\tilde{\rho}(\boldsymbol{r})}\left(R_{\boldsymbol{r}}\right)\right|-\mu_{N}^{2} \\
& =\mu_{n}+\sum_{\boldsymbol{q} \neq \boldsymbol{r}}\left|B_{\tilde{\rho}(\boldsymbol{q})}\left(R_{\boldsymbol{q}}\right)\right|\left|B_{\tilde{\rho}(\boldsymbol{r})}\left(R_{\boldsymbol{r}}\right)\right|-\mu_{N}^{2} \leq \mu_{N},
\end{aligned}
$$

by Lemma 1 and since

$$
\sum_{q \neq r}\left|B_{\tilde{\rho}(q)}\left(R_{q}\right)\right|\left|B_{\tilde{\rho}(r)}\left(R_{r}\right)\right| \leq\left(\sum_{1 \leq|q| \leq N}\left|B_{\tilde{\rho}(q)}\left(R_{q}\right)\right|\right)^{2}=\mu_{N}^{2}
$$

But

$$
\sigma_{N}^{2} \geq \int_{v_{N}^{-1}(0)}\left(v_{N}(X, \boldsymbol{\alpha})-\mu_{N}\right)^{2} d X d \boldsymbol{\alpha}=\mu_{N}^{2}\left|v_{N}^{-1}(0)\right|,
$$

whence, since $\rho(N)=N^{-1-m / n} \log N$,

$$
\left|\nu_{N}^{-1}(0)\right| \leq \sigma_{N}^{2} /\left(\mu_{N}\right)^{2} \leq 1 / \mu_{N}=O\left(\rho(N)^{-n} N^{-(n+1)}\right)=O(\log N)^{-n}
$$

so that $\left|v_{N}^{-1}(0)\right| \rightarrow 0$ as $N \rightarrow \infty$. Moreover

$$
v_{N}^{-1}(0)=\Omega \backslash \bigcup_{1 \leq|q| \leq N} B_{\tilde{\rho}(q)}\left(R_{q}\right) \supseteq \Omega \backslash \bigcup_{1 \leq|q| \leq N} B\left(R_{q} ; \rho(N)\right),
$$

whence by (10), (9) holds and so the family $\mathscr{R}$ is ubiquitous with respect to $\rho(N)=$ $N^{-1-m / n} \log N$. 
The ubiquity of the $R_{q}$ with respect to $\rho$ can be interpreted as a 'weak' Dirichlet's theorem, in the sense that given any positive integer $N$, for all but a set of points $(X, \alpha)$ of measure $O(\log N)^{-n}$ there exists a non-zero $q \in \mathbb{Z}^{m}$ such that $|q| \leq N$ and

$$
|\langle\boldsymbol{q} X-\boldsymbol{\alpha}\rangle|<\tilde{\rho}(N)=|\boldsymbol{q}| N^{-1-m / n} \log N \leq N^{-m / n} \log N .
$$

LEMMA 4. Let $\psi$ be a decreasing positive function and suppose $\lambda \geq m / n$ where $\lambda$ is the lower order of $1 / \psi$. Then

$$
\operatorname{dim} V(\psi) \geq m n+\frac{m+n}{\lambda+1} .
$$

For by $(6), \operatorname{dim} V(\psi) \geq \operatorname{dim} \Lambda(\mathscr{R} ; \varphi) \geq \operatorname{dim} \mathscr{R}+\gamma \operatorname{codim} \mathscr{R}$, where $\operatorname{dim} \mathscr{R}=m n$, $\operatorname{codim} \mathscr{R}=n$ and

$$
\gamma=\min \left\{1, \limsup _{N \rightarrow \infty} \frac{\log \rho(N)}{\log \varphi(N)}\right\}=\min \left\{1, \frac{1+m / n}{1+\lambda}\right\},
$$

since $\varphi(q)=\psi(q) /(m+1) q$. The lemma and the theorem now follow.

By modifying the arguments in $[4,6]$, it might be possible to show that the Hausdorff dimension of the set of points $X \in \mathbb{R}^{m n}$ satisfying (1) for a given additional $\alpha \in \mathbb{R}^{n}$ is $(m-1) n+(m+n) /(\lambda+1)$ when $\lambda \geq m / n$.

Denominators of the form $e^{2 \pi i k x}-1$, where $k \in \mathbb{Z} \backslash\{0\}$, occur in Fourier series arising in the study of the rotation number (see $[1, \S 12]$ ). Such expressions become very small for certain $k$, and for certain exceptional sets of 'near-resonant' $x$, they can get extremely small, causing problems with the convergence of the series. The above theorem with $m=n=1$ would apply to the corresponding exceptional sets associated with denominators involving expressions of the form $e^{2 \pi i(k x-\alpha)}-1$.

\section{Acknowledgements}

It is a pleasure to acknowledge the debt I owe Fred Chong who inspired and encouraged me while I was a student in Auckland during the late fifties. I am also grateful to the referee for pointing out some slips and obscurities.

\section{References}

[1] V. I. Arnol'd, Geometrical methods in ordinary differential equations (Springer, Berlin, 1983) (translated by J. Szücs).

[2] A. Baker and W. M. Schmidt, 'Diophantine approximation and Hausdorff dimension', Proc. London Math. Soc. 21 (1970), 1-11. 
[3] A. S. Besicovitch, 'Sets of fractional dimensions (IV): On rational approximation to real numbers', J. London Math. Soc. 9 (1934), 126-131.

[4] J. D. Bovey and M. M. Dodson, 'The Hausdorff dimension of systems of linear forms', Acta Arith. 45 (1984), 337-358.

[5] J. W. S. Cassels, An introduction to diophantine approximation (Cambridge Univ. Press, London, 1957).

[6] M. M. Dodson, 'Hausdorff dimension, lower order and Khintchine's theorem in metric Diophantine approximation', J. Reine Angew. Math. 432 (1992), 69-76.

[7] - 'Geometric and probabilistic ideas in the metrical theory of Diophantine approximation', Uspekhi Mat. Nauk 48 (1993), 77-106 (in Russian); also in Russian Math. Surveys 48 (1993), 73-102.

[8] M. M. Dodson, B. P. Rynne and J. A. G. Vickers, 'Diophantine approximation and a lower bound for Hausdorff dimension', Mathematika 37 (1990), 59-73.

[9] — - 'The Hausdorff dimension of exceptional sets associated with normal forms', J. London Math. Soc. 49 (1994), 614-624.

[10] M. M. Dodson and J. A. G. Vickers, 'Exceptional sets in Kolmogorov-Arnol'd-Moser theory', $J$. Phys. A 19 (1986), 349-374.

[11] H. G. Eggleston, 'Sets of fractional dimension which occur in some problems in number theory', Proc. London Math. Soc. 54 (1952), 42-93.

[12] V. Jarník, 'Diophantischen Approximationen und Hausdorffsches Mass', Mat. Sb. 36 (1929), 371-382.

[13] - 'Über die simultanen diophantischen Approximationen', Math. Z. 33 (1931), 503-543.

[14] M. V. Melián and D. Pestana, 'Geodesic excursions into cusps in finite volume hyperbolic manifolds', Michigan Math. J. 40 (1993), 77-93.

[15] B. P. Rynne, 'The Hausdorff dimension of certain sets arising from Diophantine approximation by restricted sequences of integer vectors', Acta Arith. 61 (1992), 69-81.

[16] W. Schmidt, 'A metrical theorem in Diophantine approximation', Canad. J. Math. 12 (1960), 619-631.

[17] W. M. Schmidt, 'Metrical theorems on fractional parts of sequences', Trans. Amer. Math. Soc. 10 (1964), 493-518.

[18] V. G. Sprindžuk, Metric theory of Diophantine approximations (Wiley, New York, 1979) (translated by R. A. Silverman).

\section{Department of Mathematics}

University of York

York YO1 5DD

UK 